\title{
THE LEFSCHETZ FIXED POINT THEOREM FOR COMPACT GROUPS
}

\author{
RONALD J. KNILL
}

\begin{abstract}
It is shown that every compact group $G$ is a $Q$-simplicial space where $Q$ is any field of characteristic zero. As a consequence it follows that $G$ satisfies a variation of the Lefschetz fixed point theorem.

It has been known for some time that the Lefschetz fixed point theorem applies to a few spaces other than just ANR spaces, especially if some care is taken to use coefficients in certain fields [2]. The case of all compact groups provides a broad class of spaces which may not have local connectivity of any order. It is shown that every compact group $G$ satisfies the Lefschetz fixed point theorem when coefficients for the homology groups are taken in a field of characteristic zero.
\end{abstract}

1. Compact groups. Suppose that $G$ is a compact group. By the analysis of A. Weil [4, pp. 88-90], $G$ is an inverse limit of compact Lie groups $G=$ proj $\lim G_{\mu}$ where each $G_{\mu}$ is locally isomorphic to a direct product of an abelian group and a semisimple group. In more detail, the connected component of the center of $G_{\mu}$ is an abelian group $A_{\mu}$ such that for a normal subgroup $S_{\mu}$ which is semisimple, $G_{\mu}=A_{\mu} \cdot S_{\mu}$ where $\mathbf{Z}_{\mu}=A_{\mu} \cap S_{\mu}$ is finite. Further $G_{\mu} \cong\left(A_{\mu} \times S_{\mu}\right) / \mathbf{Z}_{\mu}$. If $f_{\mu \nu}: G_{\nu} \rightarrow G_{\mu}$ is a homomorphism belonging to the inverse system, then $f_{\mu \nu}$ maps $A_{\nu}$ onto $A_{\mu}$ and $S_{\nu}$ onto $S_{\mu}$. Now each $S_{\mu}$ has a finite cover by a simply connected group $\tilde{S}_{\mu}$ which is a finite product of simply connected simple groups. A homomorphism from one product of simply connected simple groups onto another is just a projection of a product space onto a factor space. In summary we have the following theorem.

1. THEOREM. Let $G$ be a compact group. Then $G$ is an inverse limit of Lie groups $G=\operatorname{proj} \lim G_{\mu}$ and if $f_{\mu \nu}: G_{\nu} \rightarrow G_{\mu}$ is a homomorphism belonging to this inverse system, then there is a commutative diagram of covering compact Lie groups

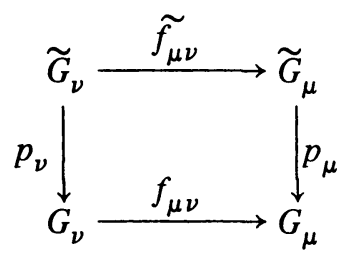

Presented to the Society, January 18, 1974; received by the editors April 18, 1974 and, in revised form, July 6, 1976.

AMS (MOS) subject classifications (1970). Primary 55C20; Secondary 22C05.

Key words and phrases. Compact group, Lefschetz fixed point theorem, $Q$-simplicial spaces.

(c) American Mathematical Society 1977 
such that the covering groups $\tilde{G}_{\nu}$ and $\tilde{G}_{\mu}$ are finite sheeted covering spaces, and such that $\tilde{f}_{\mu \nu}$ is a projection of a product space onto a factor space.

2. The $Q$-simplicial condition. Let $\alpha$ be a family of subsets of a space $X$. For a subset $B$ of $X$ let

$$
\mathrm{St}_{\alpha} B=\cup\{A: A \cap B \neq \varnothing \text { and } A \in \alpha\} .
$$

There are two simplicial complexes associated with $\alpha$. The first is the total complex, denoted $X_{\alpha}$, whose simplexes are those finite subsets $s$ of $X$ such that for some $A \in \alpha, s$ is a subset of $A$. The second is the simplicial complex called the nerve, $N(\alpha)$, of $\alpha$. Its simplexes are the finite subsets $s$ of $\alpha$ (or of the index set of $\alpha$ ) such that the set $\cap\{A: A \in s\}$ is nonempty.

The support of a chain $c$ in $C_{*}\left(X_{\alpha}\right)$ (coefficients in a field $Q$ ) is defined to be the smallest closed subset $W$ of $X$ such that $c$ is a chain of $C_{*}\left(W_{\alpha}\right)$. If $c$ is a chain in $C_{*}(N(\alpha))$, the carrier of $c$ is the smallest subfamily $\beta$ of $\alpha$ such that $c$ is a chain of $C_{*}(N(\beta))$ ( $\beta$ need not be a covering of $X$ for this to make sense). Let $\sup (c)$ be the union of those sets in the carrier of $c$.

According to Dowker [1] the two chain complexes $C_{*}\left(X_{\alpha}\right)$ and $C_{*}(N(\alpha))$ are chain homotopy equivalent; in fact his methods show that the following theorem is true.

THEOREM (DOWKER). Let $\alpha$ be a family of subsets of $X$. Then there are chain maps

$$
k^{\alpha}: C_{*}(N(\alpha)) \rightarrow C_{*}\left(X_{\alpha}\right) \text { and } l^{\alpha}: C_{*}\left(X_{\alpha}\right) \rightarrow C_{*}(N(\alpha))
$$

which are chain homotopy inverse to each other and which satisfy the two support conditions:

1. $\sup \left(k^{\alpha}(c)\right) \subset \sup (c)$, for $c \in C_{*}(N(\alpha))$,

2. $\sup \left(l^{\alpha}(c)\right) \subset \mathrm{St}_{\alpha} \sup (c)$, for $c \in C_{*}\left(X_{\alpha}\right)$.

Definition 1. A compact space $X$ is said to be " $Q$-simplicial" if for any open covering $\alpha$ of $X$ there exists an $\alpha^{\#}$ which is a finite covering of $X$ by open subsets such that $\alpha^{\#}$ refines $\alpha\left(\alpha^{\#}>\alpha\right)$ and such that for any open covering $\beta$ of $X$ there is a chain map $\omega: C_{*}\left(X_{\alpha^{*}}\right) \rightarrow C_{*}\left(X_{\beta}\right)$ such that $\sup (\omega(c)) \subset \operatorname{St}_{\alpha}(\sup (c))$, for $c \in C_{*}\left(X_{\alpha^{*}}\right)$.

This is the form of $Q$-simplicial which occurs in [2]. There is an equivalent form for nerves which we give below.

Definition 2. A compact space $X$ is said to be " $Q$-simplicial" if for any open covering $\alpha$ of $X$ there exists an $\alpha^{*}$ which is a finite covering of $X$ by open subsets such that $\alpha^{*}>\alpha$ and such that for any open covering $\beta$ of $X$ there is a chain map $\tau: C_{*}\left(N\left(\alpha^{*}\right)\right) \rightarrow C_{*}(N(\beta))$ such that $\sup (\tau(c)) \subset$ $\mathrm{St}_{\beta}\left(\mathrm{St}_{\alpha}(\sup (c))\right)$, for $c \in C_{*}\left(N\left(\alpha^{*}\right)\right)$.

Proposition. Definitions 1 and 2 are equivalent.

Proof. Let $X$ be a compact space. Let us prove that Definition 1 implies Definition 2. For an open covering $\alpha$ of $X$, let $\alpha^{*}=\alpha^{\#}$. For any open covering $\beta$ of $X$, let $\omega$ be a chain map as in Definition 1. Then let $\tau$ : 
$C_{*}\left(N\left(\alpha^{*}\right)\right) \rightarrow C_{*}(N(\beta))$ be the composition $\tau=l^{\beta} \omega k^{\alpha^{*}}$. One may compute straightforwardly that $\sup (\tau(c)) \subset \mathrm{St}_{\beta}\left(\mathrm{St}_{\alpha}(\sup (c))\right)$ for $c \in C_{*}\left(N\left(\alpha^{*}\right)\right)$. Thus $\alpha^{*}$ satisfies the conditions of Definition 2, proving 1 implies 2 .

To see that Definition 2 implies Definition 1, we again begin with an open covering $\alpha$ of $X$. By the compactness of $X$ there is an open refinement $\gamma$ of $\alpha$ such that for any subset $B$ of $X$,

$$
\mathrm{St}_{\gamma}\left(\mathrm{St}_{\gamma}\left(\mathrm{St}_{\gamma}(B)\right)\right) \subset \mathrm{St}_{\alpha}(B) .
$$

Let $\alpha^{\#}=\gamma^{*}$, where $\gamma^{*}$ is related to $\gamma$ as $\alpha^{*}$ is to $\alpha$ in Definition 2. Let $\beta$ be any open covering of $X$, and let $\beta^{\prime}$ be a common refinement of $\beta$ and $\gamma$. Let $\tau: C_{*}\left(N\left(\alpha^{\#}\right)\right) \rightarrow C_{*}\left(N\left(\beta^{\prime}\right)\right)$ be a chain map such that $\sup (\tau(c)) \subset$ $\mathrm{St}_{\beta^{\prime}}\left(\mathrm{St}_{\gamma}(\sup (c))\right)$ for any chain $c \in C_{*}\left(N\left(\alpha^{\#}\right)\right)=C_{*}\left(N\left(\gamma^{*}\right)\right)$. Let $\pi$ be the inclusion of $C_{*}\left(X_{\beta^{\prime}}\right)$ into $C_{*}\left(X_{\beta}\right)$. Then define $\omega=\pi k^{\beta^{\prime}} \tau l^{\alpha^{*}}$. Since $\pi$ and $k^{\beta^{\prime}}$ reduce supports, then for a chain $c$ in $C_{*}\left(X_{\alpha^{*}}\right)=C_{*}\left(X_{\gamma^{*}}\right)$,

$$
\left.\sup (\omega(c)) \subset \sup \left(\tau l^{\alpha^{*}}(c)\right) \subset \operatorname{St}_{\beta^{\prime}}\left(\operatorname{St}_{\gamma}\left(\operatorname{St}_{\gamma^{*}}(\sup (c))\right)\right)\right)
$$

Since $\beta^{\prime}, \gamma$ and $\gamma^{*}$ all refine $\gamma$, then by (1) and (2), sup $\omega(c) \subset \operatorname{St}_{\alpha}(\sup (c))$. This proves that $\alpha^{\#}$ satisfies the conditions of Definition 1, completing the proof of the Proposition.

The following theorem is proven in [2] in a more general form including certain noncompact spaces.

THEOREM. Suppose that $X$ is a compact space which is $Q$-simplicial. Let $f$ : $X \rightarrow X$ be a map such that the induced homology homomorphism

$$
f_{*}: H_{*}(X ; Q) \rightarrow H_{*}(X ; Q)
$$

has an image which is finite dimensional as a vector space over $Q$. Then the Lefschetz number

$$
\Lambda_{Q}(f)=\sum_{n}(-1)^{n} \operatorname{trace}\left(f_{n}\right)
$$

is defined. If $\Lambda_{Q}(f) \neq 0$ then $f(x)=x$ for some $x$ in $X$.

\section{The main theorem.}

TheOREM. Suppose that $G$ is a compact group and that $f$ is a continuous function of $G$ into itself. Let $Q$ be any field of characteristic zero. If $f_{*}$ has a finite dimensional image and $\Lambda_{Q}(f) \neq 0$, then there is a point $x$ in $G$ such that $f(x)=x$.

Proof. It suffices to show that $G$ is $Q$-simplicial. We use Definition 2 . From the first section $G$ is a projective limit of compact Lie groups $G=$ proj $\lim G_{\mu}$. Any Lie group is triangulable so assume triangulations are given compatible with the homomorphisms of the inverse system. Let $\nu>\mu$ be indices of this system. Then there is a commutative diagram of onto simplicial maps 


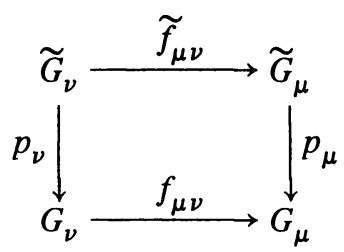

such that $\left(\tilde{G}_{\nu}, p_{\nu}, G_{\nu}\right)$ and $\left(\tilde{G}_{\mu}, p_{\mu}, G_{\mu}\right)$ are finite sheeted covering spaces and such that $\tilde{f}_{\mu \nu}$ is a projection of a product space onto a factor space. Define a chain map $\tau_{\nu \mu}: C_{*}\left(G_{\mu}\right) \rightarrow C_{*}\left(G_{\nu}\right)$ as a composition of chain maps $\tau_{\nu \mu}=p_{\nu} \tau_{1} \tau_{2}$ where $\tau_{1}: C_{*}\left(\tilde{G}_{\mu}\right) \rightarrow C_{*}\left(\tilde{G}_{\nu}\right)$ is any simplicial approximation to a cross section $\tilde{G}_{\mu} \rightarrow \tilde{G}_{\nu}$, and $\tau_{2}: C_{*}\left(G_{\mu}\right) \rightarrow C_{*}\left(\tilde{G}_{\mu}\right)$ is defined by $\tau_{2}(s)=n^{-1} \sum_{i=1}^{n} s_{i}$ where $s$ is an oriented simplex of $G_{\mu}$ and where $s_{1}, \ldots, s_{n}$ are the oriented simplexes of $\tilde{G}_{\mu}$ which cover $s$. The number $n$ is the same for every simplex, and it is clear that $\tau_{2}$ is a chain map. Since $\tilde{f}_{\mu \nu} \tau_{1}=$ identity, and $p_{\mu} \tau_{2}=$ identity, then

$$
f_{\mu \nu} \tau_{\nu \mu}=\text { identity of } G_{\mu} \text {. }
$$

It remains to show that $G$ is $Q$-simplicial. Let $G_{\mu}^{(m)}$ be the $m$ th barycentric derived complex of $G_{\mu}$. If $v$ is a vertex of $G_{\mu}^{(m)}$, then let $\operatorname{St}(v)$ be the star of $v$ in $\left|G_{\mu}^{(m)}\right|$. Let $f_{\mu}: G \rightarrow G_{\mu}=\left|G_{\mu}^{(m)}\right|$ be the limit homomorphism, and let

$$
\alpha(\mu, m)=\left\{f_{\mu}^{-1}(\operatorname{St}(v)): v \text { is a vertex of } G_{\mu}^{(m)}\right\} .
$$

This is the inverse image under $f_{\mu}$ of the star covering of $\left|G_{\mu}^{(m)}\right|$ and as such is an open covering of $G$. The family of all such coverings (as $\mu$ and $m$ range over their possible values) is cofinal in the directed set of all open coverings of $G$. Furthermore the nerve $N(\alpha(\mu, m))$ is naturally identified with $G_{\mu}^{(m)}$. Let $c$ be a chain in $C_{*}(N(\alpha(\mu, m)))$, and write $v \in c$ if $v$ is a vertex of a simplex $s$ which occurs with nonzero coefficient. If we regard $v$ as a vertex of $G_{\mu}^{(m)}$, then $\mathrm{St}(v)$ makes sense, and

$$
\sup (c)=f_{\mu}^{-1}\left(\bigcup_{v \in c} \operatorname{St}(v)\right) .
$$

It is clear that $\tau_{\nu \mu}$ induces a chain map $\tau_{\nu \mu}^{(m)}: C_{*}\left(G_{\mu}^{(m)}\right) \rightarrow C_{*}\left(G_{\nu}^{(m)}\right)$; further

$$
\sup \left(\tau_{\nu \mu}^{(m)}(c)\right)=\sup (c) \text {. }
$$

This is so, since by (3), $\sup (c)=\sup \left(f_{\mu \nu} \tau_{\nu \mu}^{(m)}(c)\right)$ and $f_{\mu \nu}$ does not change supports (by (4) and the equality $f_{\mu}=f_{\mu \nu} f_{\nu}$ ).

For an open covering $\alpha$ of $G$, we must show how to choose $\alpha^{*}$ satisfying the conditions of Definition 2. Choose any $\mu$ and $m$ such that $\alpha(\mu, m)$ refines $\alpha$, and let $\alpha^{*}=\alpha(\mu, m)$. For any open covering $\beta$ of $G$ we must define a chain map $\tau: C_{*}\left(N\left(\alpha^{*}\right)\right) \rightarrow C_{*}(N(\beta))$ such that $\sup (\tau(c)) \subset \mathrm{St}_{\beta} \mathrm{St}_{\alpha}(\sup (c))$. In fact we shall show how to define $\tau$ so that the stronger statement (5) holds.

$$
\sup (\tau(c)) \subset \mathrm{St}_{\beta}(\sup (c)) .
$$

Let $\nu$ and $n$ be such that $n \geqslant m, \nu>\mu$, and $\alpha(\nu, n)>\beta$. Define $\tau$ as the composition

$$
\tau=\pi \circ \mathrm{Sd} \circ \tau_{\nu \mu}^{(m)}
$$


where $\pi: C_{*}(N(\alpha(\nu, n))) \rightarrow C_{*}(N(\beta))$ is induced by a projection of coverings, and $\mathrm{Sd}$ is the subdivision chain map

$$
\begin{array}{cc}
\text { Sd: } C_{*}\left(G_{v}^{(m)}\right) & C_{*}\left(G_{\nu}^{(n)}\right) \\
\| & \| \\
C_{*}(N(\alpha(\nu, m))) & C_{*}(N(\alpha(\nu, n)))
\end{array}
$$

Since $\tau_{\nu \mu}^{(m)}$ and Sd preserve supports and $\pi$ satisfies $\sup (\pi(c)) \subset \operatorname{St}_{\beta}(\sup (c))$, then (4) holds. This completes the proof of the theorem.

CONCLUDING COMMENT. It would seem that any compact coset space $G / M$ of a compact group $G$ by a closed subgroup $M$ would satisfy the Lefschetz fixed point theorem, but this question remains open.

\section{BIBLIOGRAPHY}

1. C. H. Dowker, Homology groups of relations, Ann. of Math. (2) 56 (1952), 84-95. MR 13, 967.

2. R. J. Knill, Q-simplicial spaces, Illinois J. Math. 14 (1970), 40-51. MR 41 \#2664.

3. S. Lefschetz, Algebraic topology, Amer. Math. Soc. Colloq. Publ., vol. 27, Amer. Math. Soc., Providence, R. I., 1942. MR 4, 84.

4. A. Weil, L'intégration dans les groupes topologiques et ses applications, Actualités Sci. Indust., no. 869, Hermann, Paris, 1940. MR 3, 198.

Department of Mathematics, Tulane University, New Orleans, Louisiana 70118 